\title{
Financial Risk Management Via Multi Model Inference GRID Applications
}

\author{
Christian T. Brownlees* \\ University of Florence, Department of Statistics "G. Parenti" \\ E-mail: ctbeds.unifi.it
}

\section{Simone Contini}

E-mail: s.contini@oltrelinux.com

\author{
Riccardo Di Meo \\ Egrid Team \\ E-mail: dimeo@democritos.it
}

\author{
Valerio Sullo \\ ARCA SGR \& University of Florence, Department of Statistics “G. Parenti” \\ E-mail: sullo@ds.unifi.it
}

\begin{abstract}
A pivotal component of modern risk management is the employment of dynamic statistical models to forecast risk and return of financial assets. These forecasts are the fundamental ingredient on the basis of which the decisions of financial agents are made.

As the degree of complexity of these models can be quite high, in recent years the software industry has developed automated systems which provide periodic forecasts from some "benchmark" models which are ready to use for financial practitioners. Although very popular in financial consulting firms, these products are not useful for more advanced analysis and research as a consequence of the static nature of the modelling approach: forecasts are usually produced from one model which is claimed to be optimal on the basis of often not very sound statistical and economic considerations.

A much more appealing software infrastructure for financial researchers and practitioners would be one capable of providing an interactive modelling approach. By interactive modelling we mean a software environment which allows users to define which models to use, share the empirical evidence drawn from the different models of the community of users and combine different forecasts or choose the optimal one in order to achieve superior predictive ability. Recent developments in statistical Multi Model Inference (MMI) and GRID computing make this type of applications feasible.

This work presents the implementation of an MMI-GRID application which aims at reaching these goals in the context of volatility analysis and forecasting.
\end{abstract}

Grid Technology for Financial Modelling and Simulation

3-4 February 2006

Palermo, Italy

* Speaker. 


\section{Introduction}

A pivotal component of modern risk management is the employment of dynamic statistical models to forecast risk and return of financial assets. These forecasts are the fundamental ingredient on the basis of which the decision of financial agents are made. It is customary in the financial software industry to provide services consisting of the periodic delivery of forecasts produced by some "benchmark" model. One limitation of these services is that optimality of the benchmark model used can be easily put into question.

One of the frontiers of theoretical statistical research in recent years has been the development of methods which allow researchers to merge the empirical evidence which can be drawn from different statistical models. This important branch of statistical theory is referred to as Multi Model Inference (MMI) ([1]). The development of MMI methods makes it possible to develop statistical software that fully exploits the potential of a GRID infrastructure. In addition to share data and computational resources, MMI-GRID software applications allow financial researchers and practitioners to share and combine the empirical evidence which emerges from their statistical analysis without having to stick to some benchmark model which is set a priori.

The present work illustrates the implementation of an MMI-GRID application which aims at reaching these goals in context of univariate volatility analysis and forecasting. The paper is structured as follows. In Section 2 we introduce the class of Multiplicative Error Models (MEM) which can be used to forecast market volatility. Section 3 describes the multi model problem. In Section 4 we illustrate how the MMI-GRID application works. Concluding remarks follow in Section 5.

\section{Multiplicative Error Models}

The Multiplicative Error Model (henceforth MEM) is a generalisation of the ARCH family of models proposed in [2] for the modelling of non-negative time series processes, in particular financial volatility. Let the time series $\left\{x_{t}\right\}_{t=1}^{T}$ be a series of observations of some proxy or estimate of volatility, and assume that the series can be modelled by a MEM process. It is not our concern which measure of volatility is used as the methods presented are broadly applicable. Suggested series would however be realised volatility series or at least squared returns. Let $\mathscr{F}_{t-1}$ be the $\sigma$ algebra generated by $\left\{x_{1}, \ldots, x_{t-1}, z_{1, t}, \ldots, z_{r, t}\right\}$, the past history of the process and a set of $r$ weekly exogenous variables. The MEM process is defined as

$$
x_{t}=\mu_{t} \varepsilon_{t} \quad \varepsilon_{t} \mid \mathscr{F}_{t-1} \sim \operatorname{Gamma}(\phi, 1 / \phi)
$$

where, conditionally on $\mathscr{F}_{t-1}, \mu_{t}$ is the conditionally deterministic component of the process and $\varepsilon_{t}$ is an i.i.d. innovation process term. The specification of the conditional mean $\mu_{t}$ of a $\operatorname{MEM}(p, q)$ with $r$ weakly exogenous variables is

$$
\mu_{t}=\omega+\sum_{i=1}^{p} \alpha_{i} x_{t-i}+\sum_{i=1}^{q} \beta_{i} \mu_{t-i}+\sum_{i=1}^{r} \eta_{i} z_{i, t}
$$

There are many estimands which are associated with a MEM which are of interest in the analysis of the financial markets. The most obvious one is the $\tau$-step ahead conditional mean, as it 
is used to forecast the future realisation of the process

$$
\mu_{T+\tau \mid T}=\omega+\sum_{i=1}^{p} E\left(x_{t+\tau-i} \mid T\right)+\sum_{i=1}^{q} \mu_{t+\tau-i \mid T}+\sum_{i=1}^{r} z_{i, T+\tau}
$$

Other interesting estimand would be measures of persistence of the process such as

$$
\operatorname{per}=\sum_{i=1}^{p} \alpha_{i}+\sum_{i=1}^{q} \beta_{i}
$$

As the list of interesting estimand is long, in the following discussion we will just indicate a generic estimand of interest as $g$.

\section{MMI Framework for Multiplicative Error Models}

Let $\left\{x_{t}\right\}_{t=1}^{T}$ be a series of volatility measures whose dynamic is captured by a MEM. Assume that there is a group of researchers that are interested in analysing $\left\{x_{t}\right\}_{t=1}^{T}$. The researchers agree to modelling the volatility measures with a MEM, but each researcher has different opinions about which specification would be more appropriate. Furthermore, assume that each researcher has a certain focus estimand of interest $g$ which can be obtained by any MEM, and which does not have to be the same for each researcher. Assume that all the models proposed by a group of researchers lie between two extreme model specifications: the narrow or a wide model specification. In formulae, let the narrow specification be a $\operatorname{MEM}(p, q)$ with $r$ weakly exogenous variables

$$
\mu_{t}=\omega+\sum_{i=1}^{p} \alpha_{i} x_{t-i}+\sum_{i=1}^{q} \beta_{i} \mu_{t-i}+\sum_{i=1}^{r} \eta_{i} z_{i, t}
$$

and let the wide specification be $\operatorname{MEM}\left(p+\Delta_{p}, q+\Delta_{q}\right)$ with $r+\Delta_{r}$ weakly exogenous variables

$$
\mu_{t}=\omega+\sum_{i=1}^{p+\Delta_{p}} \alpha_{i} x_{t-i}+\sum_{i=1}^{q+\Delta_{q}} \beta_{i} \mu_{t-i}+\sum_{i=1}^{r+\Delta_{r}} \eta_{i} z_{i, t}
$$

A generic researcher's preferred model would be a sub model of the wide specification which nests the narrow specification. Let $p^{\prime} \in\left\{p, \ldots, p+\Delta_{p}\right\}, q^{\prime} \in\left\{q, \ldots, q+\Delta_{q}\right\}$ and $S_{r} \subset\left\{r+1, \ldots, r+\Delta_{r}\right\}$. We can than use the ordered triple $S=\left(p^{\prime}, q^{\prime}, S_{r}\right)$ to denote the generic sub model. Therefore, there is a maximum of $\left(1+\Delta_{p}\right)\left(1+\Delta_{q}\right) 2^{\Delta_{r}}$ model specifications which can be used to construct an estimate of the focus estimand of interest $g$. This implies that even if each individual model requires moderate computational resources to be estimated, the set of candidate models of all assets rapidly becomes very big. The estimators which the researchers are interested in constructing in this setting vary from $\hat{g}_{\text {wide }}$ to $\hat{g}_{\text {narr }}$, and the generic sub model estimator is $\hat{g}_{S}$ with $S \in\left(p^{\prime}, q^{\prime}, S_{r}\right)$.

The Frequentist Model Averaging (FMA) results of [3] provide the tools needed to merge empirical evidence which can be drawn from the different models in this framework. More details on the statistical aspects and finite sample performance of this machinery can be found [4]. 


\section{A MMI-GRID Application}

The purpose of the MMI-GRID application is to build up an environment where different users are allowed to interact in their analysis and to share and use the communities' empirical results. The MMI-GRID application acts both as a server and as a client. As a server the application manages user analysis instructions, as a client it submits jobs to the GRID which contain the numerical intensive parts of the users' analysis instructions. Users can connect through some client to the MMI-GRID application to perform a set of operations related to the analysis of the data of interest. These operations are analysis queries and modelling strategy instructions. By analysis queries we refer to the possibility of viewing which are the different analysis results obtained by the community. By modelling strategy instructions we mean that users can define how they want the data to be analysed. This includes specifying some new model specification which is regarded as useful for the purposes of the analysis and defining some model selection or model averaging scheme on some set of models to produce forecasts for the parameter of interest. The server side of the MMIGRID application has been built implementing an XML-RPC server in Python. The clients of the MMI-APP are simple web XML-RPC clients programmed in PHP. All the modelling strategy instructions are stored in XML database. As a client the MMI-GRID application submits jobs to the GRID containing the numerical intensive bits of the jobs. Whenever new data is available, say once a day, the MMI-GRID application translates the user instruction in GRID jobs, which are then submitted on the GRID. Once the job has been executed, the results are stored by the MMI-GRID application and made available to users. All the number crunching is performed by specific binary applications which have been optimised for numerical statistical analysis (see [4]).

\section{Conclusion}

There are some interesting aspects of this application which we think worthwhile emphasising. First we believe that sharing and merging statistical analysis is an important frontier of scientific analysis in the non experimental sciences. Why does the community of researchers and practitioners have to analyse each day individually the same data with the same models? A second interesting aspect of our application is that it allows users to benefit from a GRID infrastructure without having to know how the GRID works. More generally the user interface can be made as easy as required by the target user, so that all the problems linked to the introduction of new technologies is bypassed.

\section{References}

[1] [Burnaham \& Anderson, 2002] Burnham, K. P. \& Anderson, D. R. 2002, Model Selection and Multimodel Inference, Springer, New York.

[2] [Engle, 2002] Engle, R. F. 2002, 'New frontiers for ARCH models', Journal of Applied Econometrics 17, 425-446.

[3] [Hjorth \& Claeskens, 2003] Hjort, N. L. \& Claeskens, G. 2003, 'Frequentist model average estimators', Journal of the American Statistician Association 98, 879-899.

[4] [Brownlees, 2005] Brownlees, C. T. (2005) Multi Model Inference for Conditionally Heteroschedastic Dynamic Models, unpublished manuscript. 